\title{
AIAA 2001-1290
}

\section{SIMULATION OF TRANSONIC LIMIT CYCLE OSCILLATIONS USING A CFD TIME-MARCHING METHOD}

L. Tang

ZONA Technology, Inc., Scottsdale, AZ

R.E. Bartels

NASA Langley Research Center, Hampton, VA

P.C. Chen

ZONA Technology, Inc., Scottsdale, AZ

D.D. Liu

Arizona State University, Tempe, AZ

$42^{\text {nd }}$ AIAA/ASME/ASCE/AHS/ASC Structures, Structural Dynamics, and Materials Conference \& Exhibit

$$
\begin{gathered}
\text { 16-19 April } 2001 \\
\text { Seattle, WA }
\end{gathered}
$$

For permission to copy or to republish, contact the copyright owner named on the first page. For AIAA-held copyright, write to AIAA Permissions Department, 1801 Alexander Bell Drive, Suite 500, Reston, VA 20191-4344. 


\title{
SIMULATION OF TRANSONIC LIMIT CYCLE OSCILLATIONS USING A CFD TIME-MARCHING METHOD
}

\author{
L. Tang* \\ ZONA Technology, Inc., Scottsdale, Arizona 85251-3540 \\ Tel (480) 945-9988 / Fax (480) 945-6588 / email: tangl@zonatech.com \\ and \\ R.E. Bartels \\ NASA Langley Research Center, Hampton, VA 23681-0001 \\ Tel (757) 864-2813 / Fax (757) 864-8678 /email: r.e.bartels@larc.nasa.gov \\ and \\ P.C. Chen ${ }^{\ddagger}$ \\ ZONA Technology, Inc., Scottsdale, Arizona 85251-3540 \\ Tel (480) 945-9988 / Fax (480) 945-6588 / email: info@zonatech.com \\ and \\ D.D Liu \\ Arizona State University, Tempe, AZ 85287-6106 \\ Tel (480) 965-4117 / Fax (480) 965-1384 / email: danny.liu@asu.edu
}

\begin{abstract}
CFD-based aeroelastic computations are performed to investigate the effect of nonlinear aerodynamics on transonic LCO (Limit Cycle Oscillations) characteristics of a two-dimensional supercritical wing with the NLR 7301 section. It is found that the presentation of the viscous effects, including turbulence modeling, plays an important role on the accurate prediction of shock and LCO; and a small initial perturbation appears to produce large amplitude LCO at small mean pitch angle and plunge while a large amplitude initial perturbation produces small (or negligible) amplitude LCO at larger mean values. Also addressed in the paper is the issues related to multiblock MPI (Message Passing Interface) parallel computation.
\end{abstract}

\section{Introduction}

LCO has been a persistent problem on several current fighter aircraft and is generally encountered with external store configurations. Denegri [1] provided a detailed description of the aircraft/store LCO phenomenon. Norton [2] gave an excellent overview of LCO of fighter aircraft carrying external stores and its sensitivity to store carriage configuration and mass properties.

LCO can be characterized as sustained periodic oscillations which neither increase nor decrease in amplitude over time for a given flight condition. Using an $s$-domain unsteady aerodynamic model of the aircraft and stores, Chen, Sarhaddi and Liu [3] have

\footnotetext{
* Engineering Specialist. Member AIAA.

$\dagger$ Aerospace Engineer. Senior Member AIAA.

$\$$ Vice President. Member AIAA.

$\S$ Professor. Associate Fellow AIAA.

Copyright $(\mathcal{C} 2001$ by ZONA Technology, Inc. Published by the American Institute of Aeronautics and Astronautics, Inc. with permission.
}

shown that wing/store LCO can be a post-flutter phenomenon whenever the flutter mode contains low unstable damping. This type of flutter mode is called a "hump mode". Since the aircraft structure usually contains structural nonlinearity such as friction damping, this amplitude-dependent friction damping can suppress the growth of amplitude, thus resulting in a steady state oscillation. This is known as the nonlinear structural damping (NSD) model of the wing/store LCO. Although not thoroughly proven through tests or numerical simulations, results of the NSD show excellent correlation with flight test LCO data of F-16 throughout subsonic and transonic Mach numbers. On the other hand, other researchers, notably Cunningham and Meijer [4], believe that the wing/store LCO is due largely to the transonic shock oscillation and shock induced flow separation, called Transonic Shock/Separation (TSS) model. Edwards has suggested the TSS model and viscous effects are two major factors that cause transonic LCO for wings. He also has studied the shock buffet phenomenon in addition to transonic LCO [5]. It should be noted that, however, there is no conflict in the NSD model and the TSS 
model in that both physical effects may contribute to LCO.

Recent renewed interest in LCO is perhaps motivated by the need to further understand the physics of LCO and the current advent of CFD methodology in aeroelasticity. There are two potential computational methods for LCO prediction/investigation: the CFL $3 D$ code (version 6) [6-8] developed and supported by NASA/Langley and the POD/ROM EigenMode approach [9] originated by Dowell and Hall of Duke University. The former is a conventional time-domain CFD method whereas the latter a frequency-domain CFD method, using aerodynamic eigenmodes.

The present study plans to use a CFD time-marching method, CFL3D v6, to numerically investigate transonic LCO of a supercritical airfoil under a plunging/pitching spring-mounting system [10-12]. It is reasonable to start from investigating a twodimensional LCO case in order to better understand the physics of LCO. However, because of the complexity of a two-dimensional LCO experimental test, there is few experimental data available for comparison. According to our best knowledge, the experimental work performed by Schewe et. al. [10-12] is perhaps the only two-dimensional LCO experimental test available in documents. Those test data were immediately used by Platzer et. al. to validate their thin-layer Navier-Stokes aeroelastic solver [13-14]. While the emphasis of [13-14] was on the predictive capability of the thin-layer Navier-Stokes aeroelastic solver, our emphasis here is to investigate the effect of nonlinear aerodynamics on transonic LCO of the supercritical airfoil, such as the impact of the viscous terms, different turbulence modeling, and the initial disturbances. Also addressed in the paper is the issues related to multiblock MPI parallel aeroelastic computation.

\section{Numerical Methodology}

The computer code used in this study is CFL3D v6, which solves the three-dimensional thin-layer Reynolds averaged Navier-Stokes equations with an upwind finite volume formulation [6]. A two-dimensional problem can be calculated by using two identical grid planes, created by duplicating the two-dimensional grid.

The code uses formally third-order upwind-biased spatial differencing for the inviscid terms with flux limiting in the presence of shocks. Either fluxdifference splitting or flux-vector splitting is available. The flux-difference splitting method of Roe [15] is employed in the present computations to obtain fluxes at cell faces. Viscous terms are discretized with second- order central differencing. There are two types of time discretization available in the code. The first-order backward time differencing is used for steady calculation while the second-order backward time differencing with $\tau$-TS subiterations is used for static and dynamic aeroelastic calculation. Furthermore, grid sequencing for steady state and multigrid and local pseudo-time stepping for time marching solutions are employed. Also available in the code are many turbulence models, although here only the SpalartAllmaras model [16] and Baldwin-Lomax model [17] with the Degani-Schiff modification have been used. A detailed description of the methodology of the code can be found in [6].

One of the important features of the CFL3D code is its capability of solving multiple zone grids with one-toone connectivity. Spatial accuracy is maintained at zone boundaries, although subiterative updating of boundary information is required. Coarse-grained parallelization using the MPI protocol can be utilized in multiblock computations by solving one or more blocks per processor. When there are more blocks than processors, optimal performance is achieved by allocating an equal number of blocks to each processor. As a result, the time required for a CFD-based aeroelastic computation can be dramatically reduced. In this paper, both single and multiblock MPI parallel aeroelastic computations near the onset of flutter LCO are compared with experiment and with other computations. Figure 1 shows a C-type grid with 273 x 93 mesh points around the NLR 7301 airfoil that has been divided into eight $69 \times 47$ blocks. This and a single block version of this grid are used in the computations to follow.

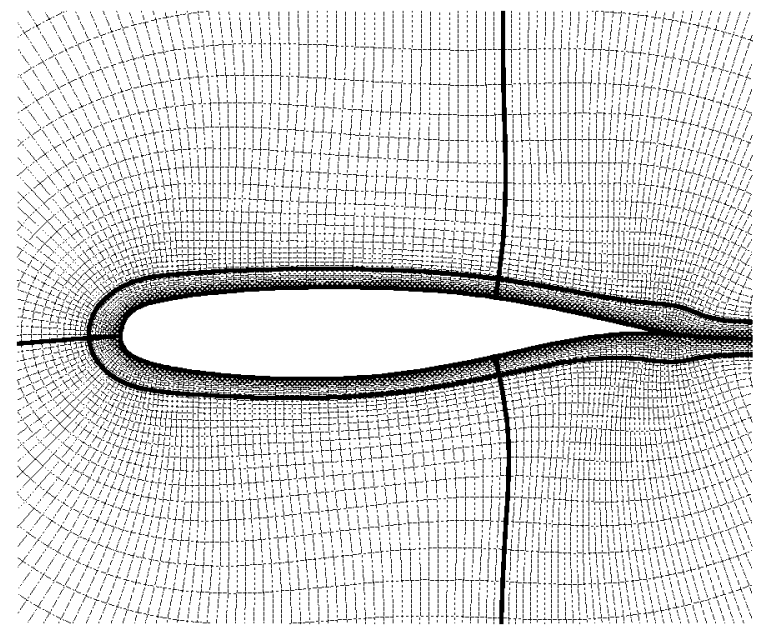

Figure 1 Multiblock C-type grid around NLR 7301 airfoil (eight 69x47 blocks)

Accordingly the mesh deformation scheme in [7] is modified to fit multiblock grids. In [7], the mesh 
deformation uses a modified spring analogy with solid body translation/rotation of the fluid mesh near solid surfaces. Initialization of the grid deformation at each step is performed using a TFI (Transfinite Interpolation) step. The mesh interior is then smoothed and grid orientation near boundaries is preserved using the modified spring analogy. In the present implementation, the subgrid based TFI scheme of [18] has been employed for initialization at each time step. That scheme uses subgrids consisting of "slave vertices" to move both block boundaries and interiors. In some instances, in order to achieve an optimal division of grid points, it is necessary to place flow field block boundaries near a moving solid surface. An example of this is shown in Figure 1. The multiblock boundary and interior movement scheme allows the user to place block boundaries near surfaces as necessary for optimal parallelization. Boundaries interior to the fluid domain near a surface respond to the local surface motion. As the airfoil moves, block boundaries move to maintain integrity of block interfaces and the airfoil surface. User controlled input makes it possible to update the mesh using this subgrid/TFI-based scheme alone or to update with an initialization using this scheme plus additional smoothing steps. These added smoothing steps, the number of which can be defined by the user, employ the modified spring analogy scheme [7]. In the current implementation the spring analogy scheme updating the mesh interior is now written in delta formulation so that the relative orientation of the original grid is retained. The solid body rotation/translation of the fluid grid is also now performed near both solid surface and block fluid boundaries.

The time-marching simulation of the aeroelastic responses is obtained using the state transition matrix solution from $t$ to $t+\Delta t$ of the state variable representation of the decoupled modal equations [1920]. The state transition matrix based scheme is optimal in the sense that it is derived from an exact solution of the free response of the modal equations. The actual scheme uses predictor/corrector steps. The predictor step marches the structure using the solution of the modal equations at the step $n$ to get the surface deflection at the time step $n+1$. This provides the surface shape for a recomputation of the fluid mesh and the fluid domain solution at $n+1$. After a solution of the fluid domain involving multiple subiterations, the corrector step then solves the modal equations at the time step $n+l$ using the averaged generalized forces at $n$ and $n+1$.

Because the CFD and CSM meshes usually do not match at the interface, $\mathrm{CFD} / \mathrm{CSM}$ coupling requires a surface spline interpolation between the two domains.
The interpolation of CSM mode shapes to CFD surface grid points is done as a preprocessing step. Modal deflections at all CFD surface grids are first generated. Modal data at these points are then segmented based on the splitting of the flow field blocks. Mode shape displacements located at CFD surface grid points of each segment are used in the integration of the generalized modal forces and in the computation of the deflection of the deformed surface. The final surface deformation at each time step is a linear superposition of all the modal deflections.

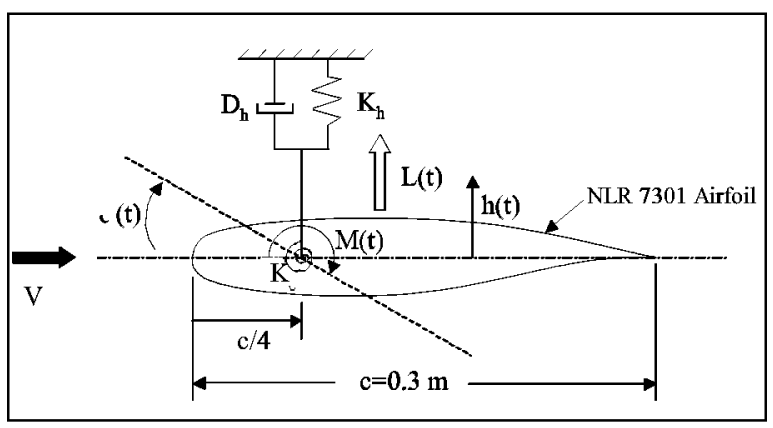

Figure 2 Two-degree-of-freedom dynamic model

The following is an account of our theoretical modeling of Schewe's experiment on transonic flutter of a twodimensional supercritical wing with an NLR7301 airfoil section [10-12]. Figure 2 depicts a simplified model of the two-degree-of-freedom test set-up. The twodimensional wing has a chord length of $0.3 m(c=0.3$ $m)$ and a span of $1 m(b=1 m)$. The pitching spring and the plunging spring are attached to the same $c / 4$ position. The corresponding two-degree-of-freedom equation of motion of the set-up reads

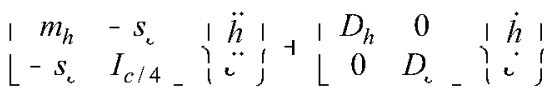

$$
\begin{aligned}
& +\left\{\begin{array}{cc}
K_{h} & 0 \\
0 & K_{e}
\end{array}\left\{\begin{array}{l}
h \\
\lfloor=
\end{array}\right\}=\left\{\begin{array}{l}
L(t) \\
M(t)
\end{array}\right\}\right.
\end{aligned}
$$

where $m_{h}$ is the total mass $\left(m_{h}=26.64 \mathrm{~kg}\right), I_{c / 4}$ is the mass moment of inertia about $c / 4\left(I_{c / 4}=0.086 \mathrm{~kg}\right.$ $\left.m^{2}\right), s_{\mathrm{L}}$ is the static unbalance $\left(s_{\mathrm{L}}=0.378 \mathrm{~kg}-\mathrm{m}\right), D_{h}$ and $D_{2}$ are the damping factors of the plunging motion $(h)$ and the pitching motion (c) respectively $\left(D_{h}=\right.$ $82.9 \mathrm{~kg} / \mathrm{s}$ and $\left.D_{2}=0.197 \mathrm{~kg}-\mathrm{m}^{2} /(\mathrm{rad}-\mathrm{s})\right), K_{h}$ and $K_{\text {. }}$ are the stiffness of the plunging spring and the pitching spring respectively $\left(K_{h}=1.21 \times 10^{6} \mathrm{~N} / \mathrm{m}\right.$ and $K_{2}=$ $\left.6.68 \times 10^{3} N-m / r a d\right)$, and $L(t)$ and $M(t)$ are the aerodynamic lift and moment respectively in Newtons. 
The aeroelastic equations and the CFD grid are maintained in dimensional form. To perform the timemarching CFD computation in CFL3D v6.0, it is necessary to convert Eq. (1) into modal coordinates, i.e.:

$$
\left\{\begin{array}{l}
h \\
c
\end{array}\right\}=14 \mid\{q
$$

where $q$ is the modal coordinate and $\psi$ is the modal matrix of the undamped structure. For this numerical example we have

$$
q=\begin{array}{cc}
1-0.1735 & 0.1004 \\
0.9277 & 3.403
\end{array}
$$

Substituting Eq. (2) into Eq. (1) and pre-multiplying the resulting equation by $q^{\mathrm{T}}$ yields

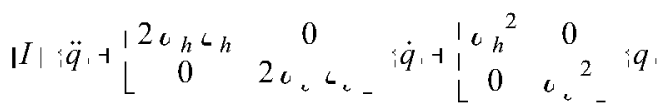

$$
\begin{aligned}
& =\psi^{T}\left\{\begin{array}{l}
L(t) \\
M(t)
\end{array}\right\}
\end{aligned}
$$

where ${ }_{h}$ and $c_{2}$ are the undamped natural frequencies of the plunging and pitching motions respectively $\left(\omega_{h}=205.4 \mathrm{rad} / \mathrm{s}\right.$ and $\left.\omega_{2}=299.5 \mathrm{rad} / \mathrm{s}\right)$, ${ }{ }_{h}$ and $c_{2}$ are the plunging and pitching damping ratios respectively $\left(c_{h}=0.00648\right.$ and $\left.c_{c}=0.00474\right)$. Note that the off-diagonal terms in the damping matrix are assumed to be zero for simplicity.

\section{Results and Discussions}

The simulated case here is the measurement No.77 documented in [12]. As mentioned before, the experimental model was a two-dimensional supercritical wing with NLR7301 section. The chord length of the wing was $0.3 \mathrm{~m}$ and the angle of attack was 1.28 . The experimental conditions were the freestream Mach number of 0.768 and the Reynolds number of $1.727,10^{6}$ based on the chord length. A transonic LCO in two-degrees-of-freedom was found at the dynamic pressure of $0.126 \mathrm{bar}$. The corresponding free-stream velocity was $254.7 \mathrm{~m} / \mathrm{s}$. The total pressure was 0.45 bar.

As shown in [13-14], because of the relatively large chord length of the airfoil with respect to the wind tunnel test section $(1 m \times 1 m)$, both the freestream Mach number and the angle of attack need to be corrected to take into account wind tunnel wall effects.
The criterion used in [13-14] was to match the computed to the measured time-averaged surface pressure distribution.

\section{Time-Averaged Surface Pressure Distribution}

First, an Euler computation is performed on a C-type grid with $293 \times 61$ points. The best agreement with the experimental data is at $M=0.734$ and $c=-0.25$. However, even for this corrected Mach number and the corrected angle of attack, the predicted shock strength is stronger than the experimental result and the location of the shock is behind the measured one, as shown in Figure 3. We have searched all Mach numbers and angles of attack. It seems impossible to match both the strength and location of the shock with Euler computation. Then two viscous computations with Baldwin-Lomax turbulence model and SpalartAllmaras turbulence model are performed on a C-type grid with $293 \times 93$ points. The corrected Mach number is found to be 0.748 for both models while the corrected angle of attack is -0.02 for the Baldwin-Lomax model and 0.15 for the Spalart-Allmaras model. Figure 3 indicates that both viscous results have a closer agreement with the experimental data, especially for shock strength and location, clearly showing that viscous effects are important for the accurate prediction of the shock.

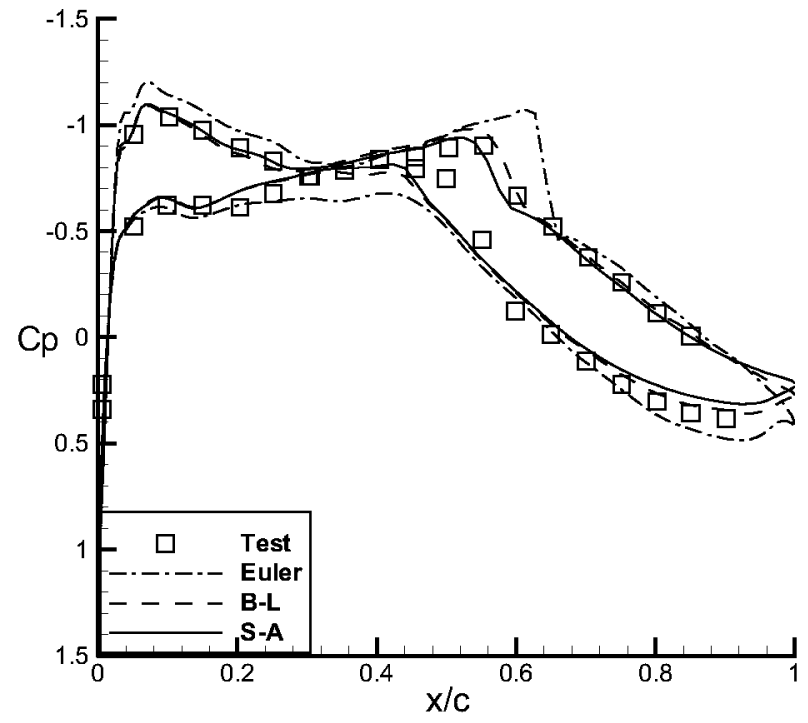

Figure 3 Time-averaged surface pressure
distribution (Aerodynamics only)

The above computed results are obtained from steady CFD analyses of a rigid two-dimensional wing surface. With taking into account the effects of elastic responses, the corrected angle of attack becomes -0.1 for Euler computation, 0.15 for the Baldwin-Lomax 
model, and 0.32 for the Spalart-Allmaras model. The corrected Mach number does not change for both Euler computation and the Baldwin-Lomax model but shifts to 0.75 for the Spalart-Allmaras model. These parameters are used in the following dynamic aeroelastic computations. Again, as shown in Figure 4, the result from Euler computation overpredicts the shock strength. The match between the computed pressures of the viscous solutions and experiment is slightly less near the shock.

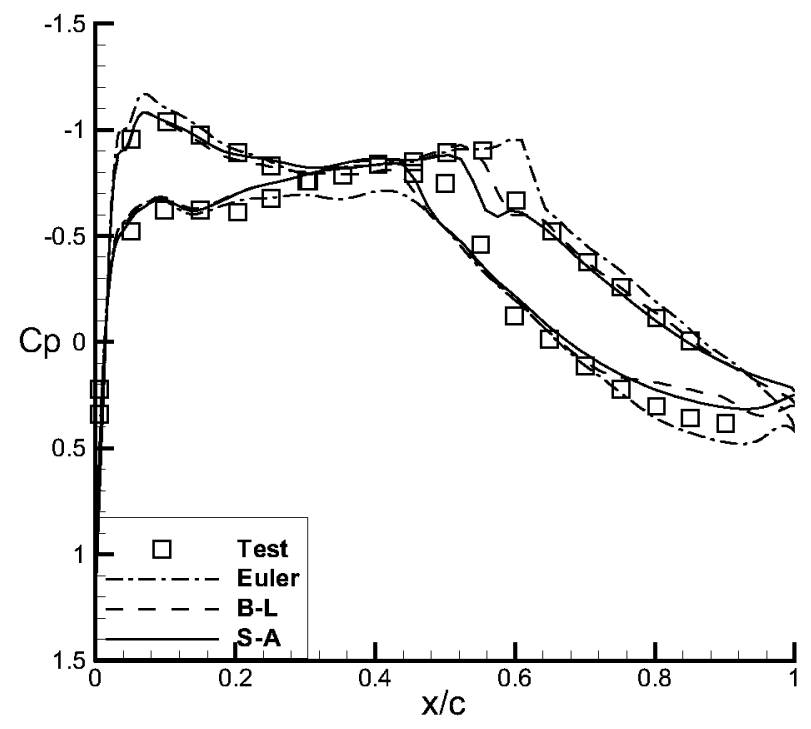

Figure 4 Time-averaged surface pressure distribution (static aeroelastic computation)

\section{Performance and Time Step Convergence}

The static aeroelastic case is used to compare run times between the single and 8 block/MPI parallel computations. Starting from a steady state a timeaccurate aeroelastic solution is marched for 800 time steps at 5 multigrid subiterations each. The SpalartAllmaras turbulence model is used in each case.

Table 1 gives performance data for the various grids, run modes, and code configurations. The first two rows in the table represent sequential computation of the 1 and 8 block versions of the grid. The 8 -block grid takes $28 \%$ less CPU time than the 1-block grid, apparently due to better caching of the smaller sized blocks. The parallel computation with 8 processors runs 7.8 times faster than the same run sequentially. The last two rows present the computational effort required for the spring analogy smoothing step and the combined TFI and aeroelasticity. Three iterations of the spring analogy scheme add about $1.5 \%$ computing time to the solution. The TFI grid movement and the aeroelastic computation add about $10 \%$ to the run time.
Table 1. Cost/Performance

\begin{tabular}{|c|c|c|c|}
\hline $\begin{array}{c}\text { Grid/comp. } \\
\text { type }\end{array}$ & TFI & $\begin{array}{c}\text { Spr. Anal. } \\
\text { Smoothing }\end{array}$ & $\begin{array}{c}\text { CPU } \\
\text { Time/ } \\
\text { Processor } \\
\text { (seconds) }\end{array}$ \\
\hline 1 block/seq & yes & yes & 8800 \\
\hline 8 block/seq & yes & yes & 5430 \\
\hline 8 block/MPI & yes & yes & 690 \\
\hline 8 block/MPI & yes & no & 680 \\
\hline 8 block/MPI & no & no & 610 \\
\hline
\end{tabular}

$\$$ also without aeroelasticity

Table 2 shows solution behavior for the single block grid for successive time step sizes. These are computed at $\mathrm{M}_{\infty}=0.753, \alpha=0.6$ and a dynamic pressure of $0.126 \mathrm{bar}$, using the Spalart-Allmaras model. Initialization is accomplished with a static aeroelastic solution, followed by an initial perturbation of the dynamic simulation of -.00114 in the velocity of the second mode. The time step sizes give 80,250 , and 800 time steps per pitch/plunge cycle. Nine subiterations per time step are used. Columns two and three are half amplitudes of the fully developed LCO plunge and pitch, while columns four and five are the plunge/pitch frequencies. Frequencies and amplitudes are computed based on a sampling of the last 8-10 cycles of motion; from this sampling the data appears to be nearly converged at the smallest time step. At the largest time step, even after 100 cycles, the amplitude slowly continued to grow while at the two smaller time steps the amplitude fully converged to LCO. In all of the remaining dynamic computations, the smallest time step size with nine multigrid subiterations is used.

Table 2. Time step convergence, 1 block grid

\begin{tabular}{|c|c|c|c|c|}
\hline$\Delta \mathrm{t}$ & $\mathrm{a}_{\mathrm{h}}(\mathrm{m})$ & $\mathrm{a}_{\alpha}($ Deg. $)$ & $\omega_{\mathrm{h}}(\mathrm{Hz})$ & $\omega_{\alpha}(\mathrm{Hz})$ \\
\hline 0.128 & .0112 & 3.72 & 34.5 & 34.5 \\
\hline 0.040 & .00917 & 3.24 & 34.3 & 34.3 \\
\hline 0.0125 & .00899 & 3.17 & 34.3 & 34.3 \\
\hline
\end{tabular}

The speed increase offered by computing in parallel is appealing. There are trade offs of course when using coarse grain parallelization. Depending on the block splitting and problem, the multiblock computations can require substantially more subiterations. This fact is most evident in the problem at hand. Figures 5 and 6 show a comparison between single block and multiblock limit cycle plunge and pitch. As an aside, it must be stated that after several hundred cycles the amplitude and frequency of the multiblock pitch and plunge had settled out and converged to values virtually identical to that of the single block grid $\left(a_{h}=0.00887\right.$ $m, \mathrm{a}_{\alpha}=3.13, \omega_{\mathrm{h}}=\omega_{\alpha}=34.3 \mathrm{~Hz}$ ). This would not be the 
case if the multiblock aeroelastic coupling and integration were not consistent with that of the single block configuration. Yet as shown in figures 5 and 6 , the early evolution of the multiblock LCO is much different than that of the single block. With these results casting some doubt on the convergence of the multiblock CFD for this LCO problem, the final computations are completed with the single block grid.

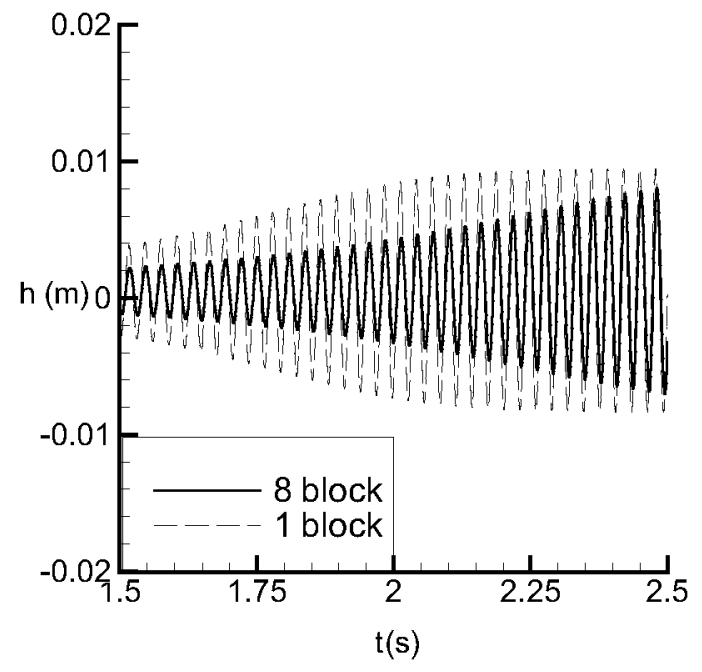

Figure 5 Plunge vs. time (N-S, small perturbation, $\mathrm{M}_{\square}=\mathbf{0 . 7 5 3 , \alpha}=0.6^{\circ}$ )

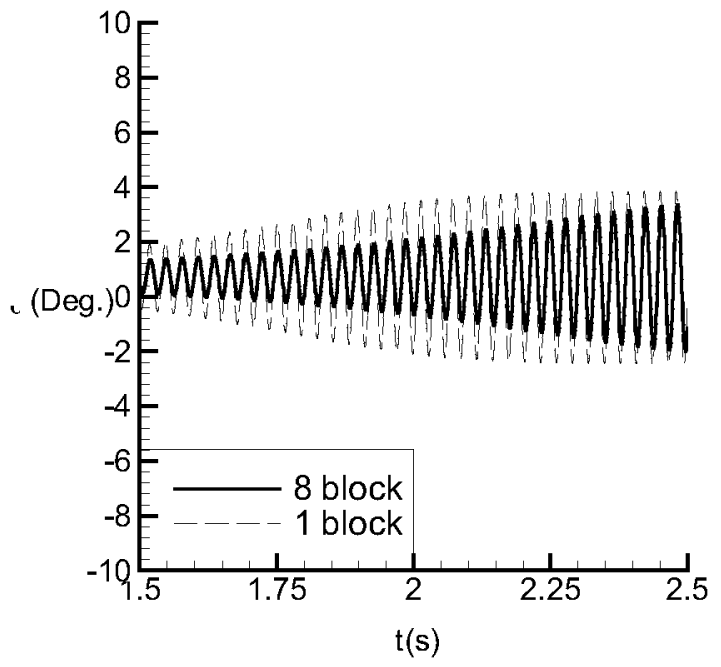

Figure 6 Pitch vs. time (N-S, small perturbation, $\left.\mathrm{M}_{\square}=\mathbf{0 . 7 5 3 , \alpha}=0.6^{\circ}\right)$

\section{Effect of Viscosity and Turbulence Model}

Figures 7 and 8 suggest that without the viscous terms, the predicted plunge and pitch modes appear never towards LCO. The effect of viscosity is to limit the amplitude of the flutter LCO. This is made clear by figures 5 and 6 and by figures 9 and 10 . Figures 9 and 10 present dynamic plunge/pitch responses to a small perturbation (-.00114 in the velocity of the second mode) using the Baldwin-Lomax and the SpalartAllmaras models. At the time of writing, the SpalartAllmaras simulation of Figures 9-10 had not reached a limit cycle whereas the Baldwin-Lomax reached large amplitude limit cycle quite rapidly. Plunge and pitch amplitudes of the B-L result are around $0.004 m$ and 2 respectively. It is clear that the turbulence model significantly alters the nature of the solution. The S-A results in each of the cases shown in figures 9-10 and figures 5-6 appear to be approaching a fixed point LCO while the B-L results of figures $9-10$ are LCO but chaotic in nature.

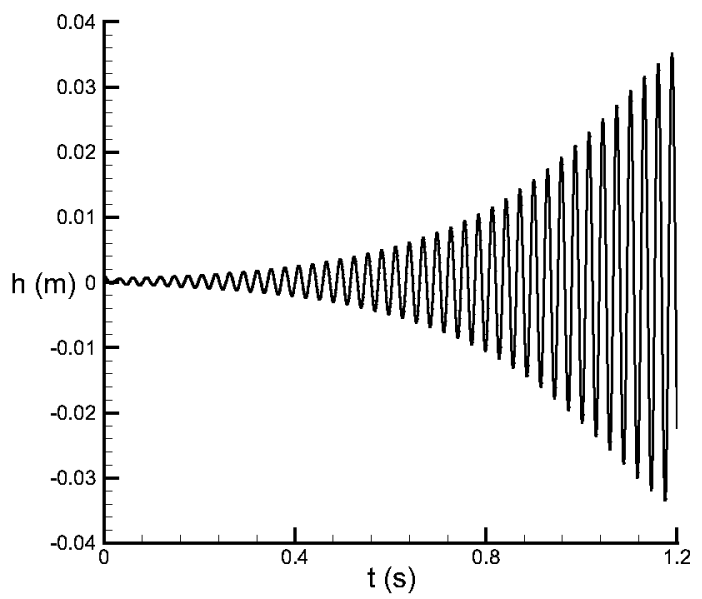

Figure 7 Plunge vs. time (Euler, small perturbation, $\left.\mathbf{M}_{\square}=\mathbf{0 . 7 3 4}, \alpha=-0.1^{\circ}\right)$

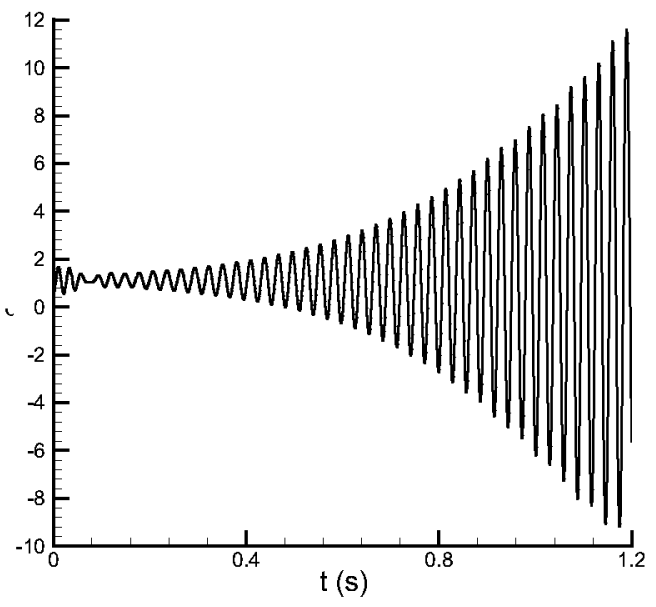

Figure 8 Pitch vs. time (Euler, small perturbation, $\left.M_{\square}=\mathbf{0 . 7 3 4}, \alpha=-0.1^{\oplus}\right)$ 


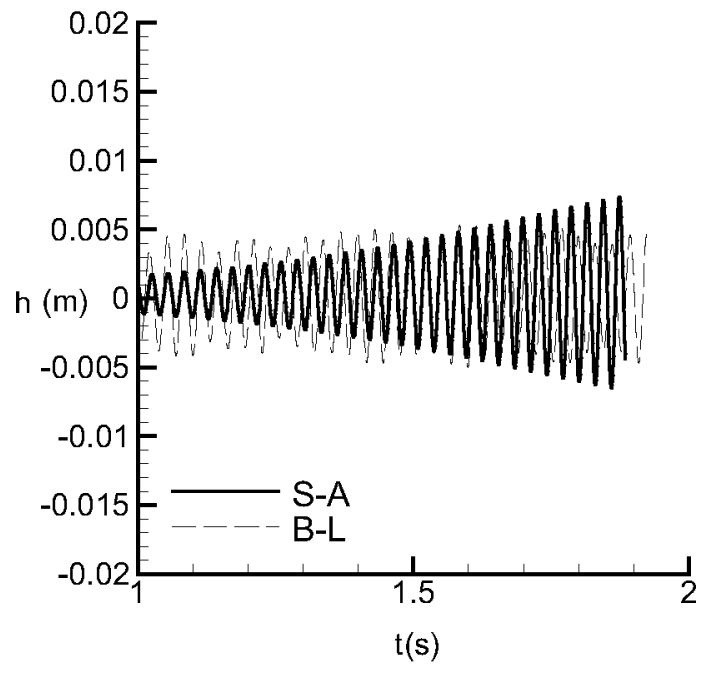

Figure 9 Plunge vs. time (N-S, small perturbation, $\mathbf{M}_{\square}=\mathbf{0 . 7 5 0}, \sqsupset=0.32^{\circ}(\mathrm{S}-\mathrm{A})$, $\left.\mathrm{M}_{\square}=\mathbf{0 . 7 4 8 ,},=\mathbf{0 . 1 5 ^ { \circ }}(\mathrm{B}-\mathrm{L})\right)$

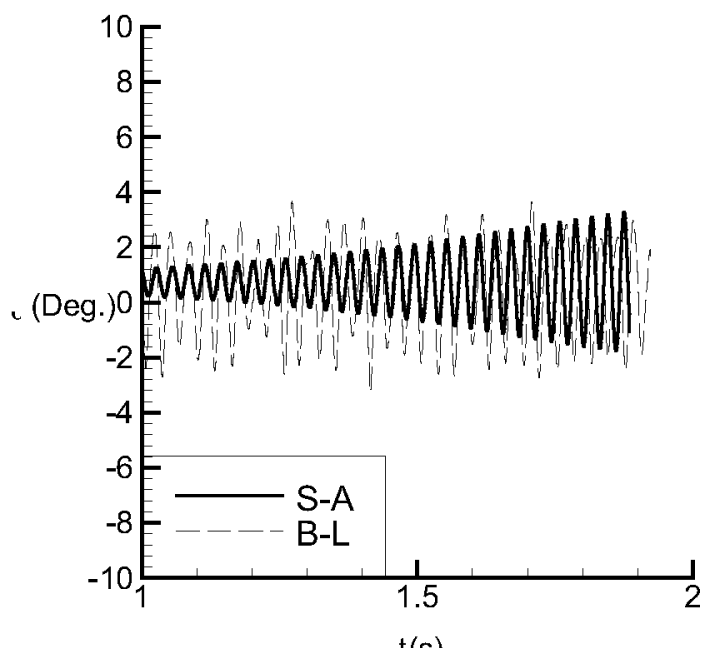

$\mathrm{t}(\mathrm{s})$

Figure 10 Pitch vs. time (N-S, small perturbation, $\mathrm{M}_{\square}=\mathbf{0 . 7 5 0}, \sqsupset=0.32^{\circ}(\mathrm{S}-\mathrm{A})$, $\left.\mathrm{M}_{\square}=\mathbf{0 . 7 4 8}, \sqsupset=\mathbf{0 . 1 5 ^ { \circ }}(\mathrm{B}-\mathrm{L})\right)$

\section{Effect of Perturbation Size}

The effect of initial perturbation size is studied by repeating earlier simulations with both turbulence models, but with a large initial perturbation (-.114 in the velocity of the second mode). Results are shown in figures 11 and 12. After the expected very large transients passed, the solutions using the two turbulence models appear to have reached LCO solutions. In response to the small perturbations both yielded mean plunge and pitch very near their static equilibrium positions. In response to the large perturbation, both the amplitude and mean plunge and pitch had offset significantly. Mean plunge had offset to values of $0.004 \mathrm{~m}$ (B-L) and $0.003 \mathrm{~m}$ (S-A). Mean pitch angles had offset to values of 3 degrees (B-L) and 1.9 degrees (S-A). Amplitudes of both turbulence model simulations are much smaller in response to the large disturbance and both show evidence of continuing chaos. The amplitudes of plunge are around $0.002 \mathrm{~m}$ (B-L) and $0.001 m$ (S-A). Amplitudes of pitch are around 1 degree (B-L) and 0.2 degree (S-A).
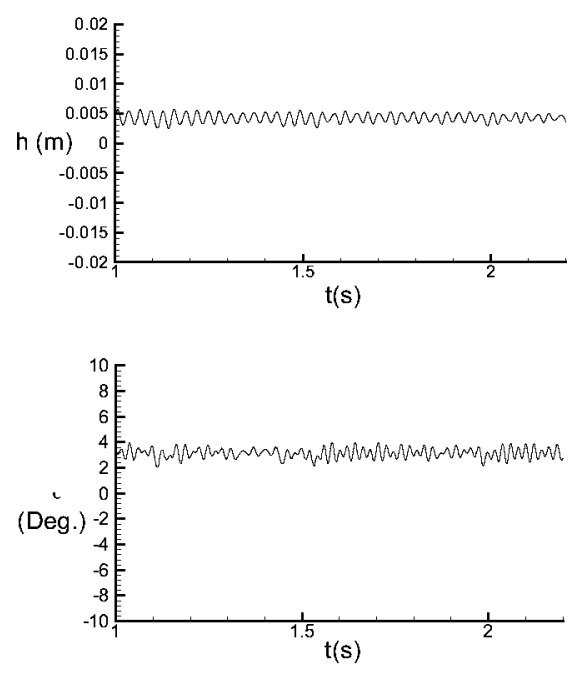

Figure 11 Plunge and pitch vs. time (B-L, large perturbation, $\mathrm{M}_{\square}=\mathbf{0 . 7 4 8 ,} \sqsupset=\mathbf{0 . 1 5 ^ { \circ }}$ )
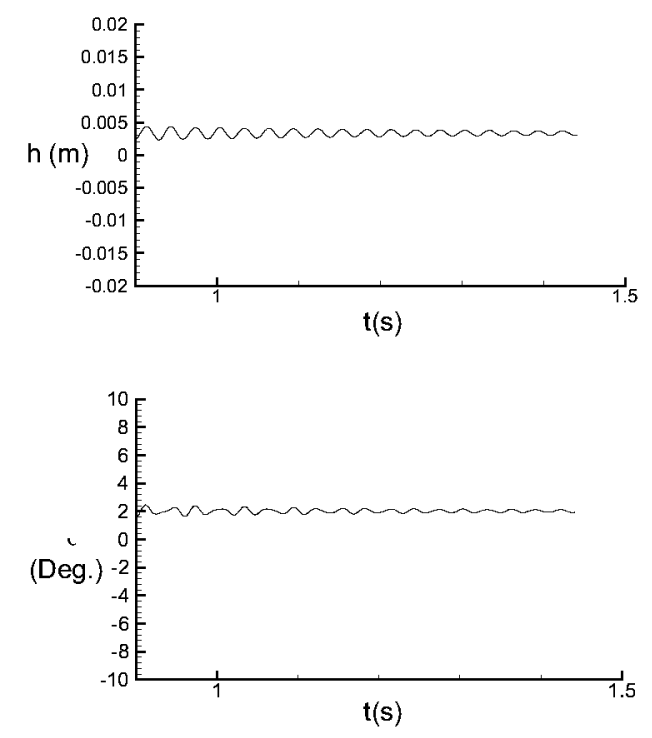

Figure 12 Plunge and pitch vs. time (S-A, large perturbation, $M_{\square}=\mathbf{0 . 7 5 0 ,} \sqsupset=0.32^{\circ}$ ) 
The plunge and pitch amplitudes computed here with the single and multiblock grids, due to small perturbation, are generally in the range of those computed elsewhere. In particular, the presently computed pitch amplitude due to the S-A model $\left(\mathrm{a}_{\square}=\right.$ 3.17 degrees) agrees very well with other results, while the plunge amplitude appears to be much lower $\left(a_{h}=\right.$ $0.009 \mathrm{~m}$ computed here). [13] These pitch amplitudes are much larger than the pitch amplitudes of the experiment. (.2-.4 degrees) The mean pitch angle at .7 degree computed here compares with a mean angle of 1.28 degrees in the experiment.

The amplitudes of responses due to the large perturbation actually are much closer to those of experiment. The pitch amplitude for the S-A model (around .2 degrees) and mean pitch (around 2 degrees) agrees quite well with experiment. It must be cautioned that the data under discussion (i.e. of figure 12) may not have reached $\mathrm{LCO}$, and may in fact be very slowly diminishing in amplitude. What can be said is that the small initial perturbation produced large amplitude LCO at small mean pitch angle and plunge while a large amplitude initial perturbation produced small (or negligible) amplitudes at larger mean values. Future computations with intermediate values of perturbation may produce somewhat larger amplitude (than the small or negligible) and lower mean pitch (than the large mean values) that produce a better match with experiment.

A final comment about the B-L results must be made. The chaotic nature of the B-L results must be taken with a certain amount of caution. To what extent the chaotic nature of the LCO is due to numerical as opposed to physically based reasons is not certain. What is remarkable is that both turbulence models produce a similar reduction in LCO amplitudes as a result of the large initial disturbance.

\section{Conclusions}

Viscous effects are found very important for the prediction of both shock and LCO. Special attention should be paid to the numerical representation of viscous effects, including turbulence modeling. A small initial perturbation appears to produce large amplitude LCO at small mean pitch angle and plunge while a large amplitude initial perturbation produces small (or negligible) amplitude LCO at larger mean values.

\section{Acknowledgement}

The authors gratefully acknowledge Dr. Schewe of DLR for his consultation on the LCO experimental setup and its structural design. They also like to thank
Prof. Platzer of NPL, Monterey, and Dr. Weber of MTU, Munich for their helps in providing us with CFD data. The ZONA Technology authors (Tang, Chen, and Liu) would like to acknowledge AFOSR (Dr. Brian Sanders/Dr. Dan Segalman are technical monitors) for its sponsorship of the present work under STTR phase I contract.

\section{References}

1. Denegri, C.M., "Limit Cycle Oscillation Flight Test Results of a Fighter with External Stores," AIAA-2000-1394.

2. Norton, W.J., "Limit Cycle Oscillation and Flight Flutter Testing," Proceedings of the Society of Flight Test Engineers $21^{\text {st }}$ Annual Symposium, 1990.

3. Chen, P.C., Sarhaddi, D. and Liu, D.D., "Limit Cycle Oscillation Studies of a Fighter with External Stores," AIAA-98-1727.

4. Cunningham Jr., A.M. and Meijer, J.J., "SemiEmpirical Unsteady Aerodynamics for Modeling Aircraft Limit Cycle Oscillations and Other Nonlinear Aeroelastic Problems," International Forum on Aeroelasticity and Structural Dynamics, Manchester, U.K., Jun. 1995.

5. Edwards, J.W., "Transonic Shock Oscillations and Wing Flutter Calculated with an Interactive Boundary Layer Coupling Method," EuromechColloquium 349 Simulation of Fluid-Structure Interaction in Aeronautics, Göttingen, Germany, Sep. 1996.

6. Krist, S.L., Biedron, R.T. and Rumsey, C.L., "CFL3D User's Manual Version 5.0," NASA Langley Research Center, Hampton, VA, 1997.

7. Bartels, R.E., "Mesh Strategies for Accurate Computation of Unsteady Spoiler and Aeroelastic Problems," Journal of Aircraft, Vol. 37, pp.521$525,2000$.

8. http://fmadwww.larc.nasa.gov/ biedron/Cfl3dv6/cfl3dv6.html

9. Hall, K.E., Thomas, J.P. and Dowell, E.H., "Reduced-Order Modeling of Unsteady Small Disturbance Flows Using a Frequency-Domain Proper Orthogonal Decomposition Technique," AIAA-99-0655.

10. Schewe, G. and Deyhle, H., "Experiments on Transonic Flutter of a Two-Dimensional Supercritical Wing with Emphasis on the Nonlinear Effects," Proceedings of the Royal Aeronautical Society Conference on Unsteady Aerodynamics, London, U.K., Jul. 1996.

11. Knipfer, A. and Schewe, G., "Investigations of an Oscillation Supercritical 2-D Wing Section in a Transonic Flow," AIAA-99-0653. 
12. Knipfer, A., Schewe, G. and Wendt, V., “ Numerische und experimentelle Untersuchungen an einem schwingenden NLR 7301-Profil in transsonischer Stroemung, Teil 1: Flattern und erzwungene Schwingungen," DLR Bericht IB 232$98 \mathrm{~J} 05,1998$.

13. Weber, S., Jones, K.D., Ekaterinaris, J.A. and Platzer, M.F., "Transonic Flutter Computations for a 2-D Supercritical Wing," AIAA-99-0798.

14. Castro, B.M., Ekaterinaris, J.A. and Platzer, M.F., "Transonic Flutter Computations for the NLR 7301 Airfoil Inside a Wind Tunnel," AIAA -2000-0984.

15. Roe, P.L., "Approximate Riemann Solvers, Parameter Vectors, and Difference Schemes," Journal of Computational Physics, Vol. 43, pp.357372, 1981.

16. Spalart, P., and Allmaras, S., "A One-Equation Turbulence Model for Aerodynamic Flows," AIAA-92-0439.

17. Baldwin, B., and Lomax, H., "Thin Layer Approximation and Algebraic Model for Separated Turbulent Flow," AIAA-78-257.

18. Hartwich, P.M., and Agrawal, S., "Method for Perturbing Multiblock Patched Grids in Aeroelastic and Design Optimization Applications," AIAA-972038.

19. Edwards, J.W., Bennett, R.M., Whitlow, W.Jr., and Seidel, D.A., "Time-Marching Transonic Flutter Solutions Including Angle-of-Attack Effects," Journal of Aircraft, Vol. 20, pp.899-906, 1983.

20. Cunningham, H.J., Batina, J.T., and Bennett, R.M., "Modern Wing Flutter Analysis by Computational Fluid Dynamics Methods," Journal of Aircraft, Vol. 25, pp.962-968, 1988. 